\title{
QUATRIÈME PARTIE - CONCLUSIONS ET ENSEIGNEMENTS
}

Les considérations qui précèdent tendaient à mettre en relief Ia genèse de la catastrophe du Gleno et les causes directes l'ayant pu déterminer ou aggrarer, ainsi que les responsabilités y engagées : ceci, au point de vue exclusivement technique. L'analyse de ses contingences permet, en outre, sous certains rapports, d'en dégager quelques autres que nous ne saurions passer sous silence ; elles sont tout à la fois d'ordre moral et administratif.

Parmi ces demières, une des plus graves réside, on l'a déjà vu, au cours de l'exposé de la construction de l'ourrage (chap. IV), dans l'absence de tout contrôle de l'Etat sur l'exécution dẹs travaux. Les Ingénieurs du Gênie Civil (1) affirment, en effet, que leur service n'avait à connaître que la conception du projet présenté par le concessionnaire, non à en contrôler ni surveiller la stricte application...

Aussi invraisemblable que cela puisse paraître, comme il ny a pas licu de mettre en doute laffirmation de nos collègues italiens, cela souligne de façon tragique, hélas ! la grave et dẻplorable lacune existant dans la législation de nos voisins en matière d'hydraulique; nous ne doutons point qu'elle soit rapidement comblée.

Lne autre raison de même ordre, non moins grave du reste, dont nous trouvons mention dans la conférence de M. Walther Schurter à la section vaudoise des Ingénieurs et Architectes, aussi bien que dans celle de notre camarade Keller à la Chambre Syndicale des Forces Hydrauliques, réside dans la facilité avec laquelle, en Italie, on octroyait, jusqu'à ces temps derniers, les concessions hydrauliques, facilité qui, de capitalistes et même d'aventuriers, en quête de spéculations, fit des concessionnaires, dont certains crurent, malheureusement, que le décret de concession leur conférait la compétence nécessaire pour l'exploitation... ainsi que cela se vit au Gleno (2).

Disons, par surcroît et dans le mème ordre d'idées, que cette facilité. n'était surpassée que par celle avec laquelle le premier venu peut encore - en Italie comme en trop d'autres pays s'improviser entrepreneur de Travaux Publics, pourvu qu'il dispose de capitaux, puisque tant est que dans notre actuclle société, si la compétence ne procure que rarement les cappitaux, par contre ces derniers procurent toujours celle-là...

C'est ainsi que notre camarade Keller a pu reconnaître, en l'un des Entrepreneurs du barrage, un individu qu'il employait comme "sergent recruteur d'hommes ", en 1910, lors de la construction de la centrale de Mazzuno (détruite par la catastrophe), C'est ainsi que nous voyous couramment, sur nos chantiers de Travaux Publics, des cantiniers s'établir "tâcherons ", puis "entrepreneurs "(l'appétit vient en mangeant...) Si, du seul point de vue social, cette pratique, essentiellement démocratique en ce sens qu'elle constitue une intéressante décentralisation des travaux et une concurrence non négligeable pour les grands " trusts " d'entreprises devenus par trop puissants et arrogants depuis la guerre, n'est point à réprouver, il faut bien reconnaịtre que, du point de vue technique, elle n'est pas sans présenter de sérieux inconvénients : l'acquisition d'une réelle capacité technicue étant, pour ces gens-là, beaucoup plus lente et difficile que celle de la "capacité financière ". Mais, si leurs appétits et prétentions dépassent trop souvent leurs capacités techniques,

(1) Administration correspondant à notre corps des Ponts et Chaussées.

(2) Bien que ses dispositions ne soient pas impossibles à " tourner ", notre législation française est, à ce point de vue, plus complète que celle de nos voisins, puisque tout dossier de demande de concession doit obligatoirement comporter l'exposé des capacités techniques et des capacités financières du demandeur. Article published by SHF and available at $n$ ttp://wwW.sht-Ihb.org or disons de suite que Sociétés privées et Administrations publiques ont, dans l'adjudication restreinte (par laquelle ne sont appeles en compétition que des Entrepreneurs connus, ayant doné leur mesure de technicité) un moyen facile d'écarter semblables prétentions inconsidérées, tout spécialement lorsq̨u'il s'agit d'ouvrages importants, comme les barrages.

\section{Enseignements à retirer de la catastrophe.}

Les causes de la catastrophe et les responsabilités y engagées nous paraissant ainsi dûment établies, nous termir er.ns celte étude - que nous voudrions aussi complète que possible - par l'indication de differents enseignements qui nous semblent se dégager nettement des circonstances layant entourée.

Implantation : Irondations. - Fn aucun cas, et sous aucun prétexte, on ne doit méconnaître ce principe f́lémentaire de l'art de construire qui veut que tout ouvrage, dans la moindre de ses parties, repose sur le terrain de fondation par des plans horizontaux; ce qui conduit inévitablement, dans le cas de barrages comme dans celui de ponts ou de viadues, à déraser en redans (ou "gradins") le terrain ou la roche supportant l'ouvrage, terrain le plus souvent incliné sur l'horizontale, et à ne jamais.s'accommoder de sa déclivité pour asseoir les massifs de fondation, mème si ces massifs doivent ètre liés à la roche par des aciers ver ticaux.

Disons enfin, qu'en tout état de cause, et dût la construction augmenter d'importance de par son déplacement, un barrage déjà sollicité plus ou moins au glissement de par sa conception, ne doit jamais, non plus, être implapté au sommet d'une forte pente longitudinale de la vallée, comme ce fut le cas an Gleno.

Nous voulons croire que ces précautions d'implantation, rigoureusement indispensables, eussent été prises si les entrepreneurs des travaux eussent été des techniciens.

Maçonneries. - L'emploi de la chaux est absolument contreindiqué pour la construction de semblables grands ouvrages ; yuoique confusément, peut-être, cela a bien élé senti au Gleno, par les constructeurs, puisqu'ils abandonnèrent ce liant pour lui substituer le ciment à la partie supérieure du soubassement (1).

Pourtant, nous sommes étrangement surpris que, s'étant arrêté à l'emploi de ce liant, on ait négligé de se le procurer dans des usines connues, livrant de bons produits; lesquelles sont pourtant nombreuses dans la Haute-Ilalie, et que, pis encore, ayant décidé d'une fabrication sur place, on ait omis, involontairement ou non, de recourir au mélange de pouzzolane, bien connu et employé en ces régions, afin de conférer, pour le moins, aux produits de la fabrication un certain indice d'hydraulicité.

Sans revenir davantage sur ce sujet, nous ajoutterons qu’à notre avis, aux impérieuses raisons techniques prohibant l'emploi do la ehaux en pareilles constructions; s'ajoutent des raisons morales non moins sérieuses : lorsqu'un ouvrage semblable constitue, en somme, une perpétuelle menace suspendue sur les installations industrielles, les biens et les personnes de toule une vallée, ce n'est rien de moins qu'un crime que de lésiner sur les moyens constructifs et de négliger d'entourer son édification de la moindre précaution destincé à accroître sa sécurité ultérieure !

(1) Substitution qui fut, du reste, inefficace, parce qu'elle ne fut pas complètée par la seule mesure - draconienne et couteuse, il est vrai - qui s'imposait : la démolition et la rélection, au ciment, de la partie déjà exćcutée à la chaux... (Il est cles économies criminelles et ruineuses $\frac{1}{3}$ ) 
Et, dans cet ordre d'idées, nous dirons encore qu'au choix du liant doit s'ajouter une sélection rigoureüse des autres matériaux, spécjalement des sables et des graviers, ainsi qu'une série d'expériences, répétées pendant toute la duréc des travaux, et portant sur la composition et le mouillage des mortiers et bétons (1), toutes précautions qui, volontairement ou non, ont encore été deplorablement négligées au Gleno.

Mise en charge. -- La mise en charge des barrages doit toujours itre pratiquée avec beaucoup de prudence et de précautions, et, s’il était possible d'établir une gradation dans cetto prudence, nous serions tenté de dire qu'elle doit aller croissant avec la hauteur et l'importance de l'ouvrage.

En tout premier lieu, elle ne saurait être effechuéc avant la jrise des mortiers ; et, d'autre part, pour etre rationnelle, elle doit être rigoureusement contrôlée par l'observation minutieuse des déformations élastiques de la structure, ainsi que des fuites auxquelles elles peuvent donner lieu.

Ici encore, nous déplorons d'avoir à constater qu'au Gleno ces indispensables précautions n'ont pas été prises, puisque la mise en charge a commencé le 2 août 1923, alors que la superstructure à arches multiples n'était pas à moitié achevée (Photos Nos $6,7,8$ ), pour être totale le 22 octobre suivant, avant même l'achèvement des parachèvements (Ploto No 9) ; et que les fuites, particulièrement abondantes, furent traitées par le mẹpris. Il nous paraît superflu de souligner que ces négligences furent d'autant plus coupables qu'avec la nature des maçonneries mises en couvre il $y$ avait lien de redoubler de prudence et de précautions. Or, il est indubilablement établi que l'ouvrage n'a pas cédé inopinément, d'un seul coup ; partant, il n'y a pas l'ombre d'un doute que des observations sérieuses, effectuées après cette imprudente mise en charge, eussent décelé l'instabilité de l'équilibre qui en résulta presque immédiatement, de même que les glissoments qui, manifestement, se sont produits avant la rupture.

Ainsi eussent ité évités, sinon la ruine du barrage - à laquelle il élail destiné par ses vices de construction, - au moins les ruines et les denils que sema la catastrophe dans l'industrieuse vallée du Dezzo.

Sélection et groupcment des responsabilités concessionnaires. - Dans tous les cas, et ce sont les plus fréquents, où un barrage comme celui qui nous occupe établit une régularisation du débit du torrent dont sont appelés à bénéficier les industriels ou usagers d'aval, il est judicieux, autant qu'équitable du reste, que ces derniers participent à sa construction.

Soucieux des intérêts de la collectivité, l'Etat, qui a charge de la coordination des aménagements hydrauliques pour éviter les dispersions d'efforts isolés, doit, le cas échéant, imposer cette parlicipation aux réfractaires. Disons aussi que son rôle doit s'etendre plus loin encore et viser plus haut; ceci non seulement dans lc choix, comme concessionnaires, de personnalités présenlant les plus sérieuses référenees techniques, mais aussi, surtont même, dans l'examen des projets que ces derniers se proposent. de réaliser, le plus souvent avec une mécomnaissance insigne des intérêts de la collectivité, qui se déjouille par trop de ses droits à leur profit exclusif.

S’i cn eût été ainsi au Gleno, il est certain quẻ l'Administration n'êt pas octroyé à lin tisserand, aussi peu préparé que possible à semblable exploitation, une concession, comportant des travaux aussi importants pour d’aussi maigres résultats à son seul

(1) Voir, a ce sujet, nos études parues dans les Annales de la Construction et dans La Vie Technique et Industrielle (Vol. II, tome II, p. 39, 1921), sur le "magesinage des ciments et le dosage mathímatique des mortiers et bútons. profit (1), alors que, trop contents de l'aubaine, les industriels d'aval, bénéficiant d'un débit accru et régularisé, se dérobaient grossierement à toute participation. Et il est non moins certain que si ces derniers eussent élé intéressés dans la construction de l'ouvrage, celui-ci n'eût pas été édifié de façon aussi déplorablẹ ct que nous n'aurions pas à déplorer la catastrophe occasionnée par sa rupture du 1 er décembre dernier.

Mesures à prendre pour déceler et éludier les mouvements éventuels des barrages. - Inexistantes sont, à notre connaissance, les dispositions prises sur les ouvrages existants -_. mème en Amérique, pays de toutes les initiatives en cette matière - pour révéler leurs mouvements éventuels, les suivre dans leur extension, les étudier et, le cas échéant, permettre de prendre à temps les mesures nécessaires pour éviter une catastrophe et sauver au moins la valiée dans le cas ou l'ouvfage ne pourrait l'être.

On se repose par trop, à notre avis, sur la rationalité de leur conception et la perfection de leur construction, pour, une fois mis en service, se dispenser de les suivre pendant l'exploitation.

L'immuabilité de ces ouvrages, généralement construits dans des régions assez tourmentées, est pourtant chose bien relative, ne fût-ce qu'en rapport avec les mouvements de l'écorce terrestre attestés journellement, de tous côtés, par les observations des stations sismologiques; et, ce seul point de vue fût-il, ce qui n'est point, le seul à envisager, il nous paraît qu'il doit y avoir quelque chose à faire pour connaître les mouvements accusés par la construction.

En particulier, et sans que cette indication soit nullement. limitative, il nous semble que l'installation de plusieurs trépidomètres, répartis sur toute la longueur du barrage, serait de nature à fournir de très intéressantes indications, que complèterait heureusement, à coup sûr, fixés en différents points de la structure, une série de niveaux à réflexion reliés à des enregistreurs de haute précision à échelle graduée amplificatrice et à des index pratiquement immobiles, de façon à relever, par diagrammes, tant les divers mouvements pouvant solliciter l'ouvrage que, dans chacun d'eux, les positions respectives des diverses membrures. Ajoutons qu'un sismographe ne serait nullement déplacé dans le poste d'observations.

Pour terminer cette étude, déjà longue, nous voudrions enfin, qu'il nous soit permis d'examiner rapidement et. en général, du seul point de vue social, les contingences de catastrophes comme celle du Gleno, essentiellement évitables, on l'a vu, si l'autorité administrative apportait plus de circonspection dans l'octroi de ses concessions, et le contrôle de leurs travaux ; les concessionnaires plus de tact dans le choix de leurs Entrepreneurs; ceux-ci plus de conscience et de compétence technique dans l'accomplissement de leurs obligations contractuelles.

En présencè de catastrophès comme celle qùe nous venons d'étudier, pour rares qu'elles soient,-- fort heureusement,-le processus des "mouvements d'opinion " est toujours le même : pendant que les technicieris ènquètent et dissertent pour en tirer les enseignements qu'elles comportent, les ignorants et les sceptiques - à la remorque dés "requins " de la finànce jouant à la baisse - vendent en masse tous les titres des industries electriques qu'ils possèdent, déterminant l'effondrement des cours, pont profiteront ces mêmes "requins ". Au milieu d'un affolement général, d'aucuns vont jusqu'à crier "au sabotage 1 ; et le grand public; ballotté entre les informations dont on l'abreuve, toutes tendancieuses en matière de bourse, est de suite conduit

(1) Résultats assez maigres, en effet, sì l'on pense que de pareils travaux furent exécutés pour la seule alimentation d'une usine de $1.500 \mathrm{Kw}$. do puissance nominale, capable seulement d'environ $850 \mathrm{Kw}$. continus. 
à se croire indignement exploilé par les Entreprises les plus solides comme les plus honorables, allant parfois jusquða accuser l'électricité elle-mème de faillite... .

Il n'y a pas lieu de généraliser aussi péjorativement. De mème qu' " une hirondelle ne fait pas le printemps ", un barrage rompu, sur des centaines, des milliers peut-être, de par le monde, qui remplissent régulièrement leur fonctlon, sans ćclat ni défaillance, ne saurait être considéré autrement que comme un incident déplorable, certes, mais toujours possible, et qui doit comporter en lui-nème une dure lecon pour l'électrotechnique en général.

De même qu'un accident de chemin de fer ou d'aviation, si terrible fut-il, ne saurait entraver un seul instant la marche en avant des transports ferroviaires ou aéronauliques, devenus indispensables à la vie mondiale d'aujourd'hui, une catastrophe comme celle du Gleno ne saurait retarder d'un seul jour la mise en valeur, déjà si en retard, de nos ressources hydrauliques, qui dort nous libérer du lourd tribut des importations de combustibles.

Mais, pour avancer à coup sûr, pour que la voie du progrès ne soit pas jalonnée de deuils et de ruines, pour qu'enfin chacun dans sa sphère contribue à aflirmer ce progrès avec une ferveur égale à la foi qu'il place en lui, il importe que de pareilles leçons, si douloureuses, ne demeurent pas lettres mortes. Il importe que les populations des régions à aménager soient mises en confiance, tant par la science de nos ingénieurs que par la sollicitude de l'Administration à leur égard, et par la rigueur de son contrôle, vis-à-vis de travaux qui constituent autant d'épées de Damoclès suspendues sur leur tête. Il importe, en outre, il importe surtout, que prenne fin, en matière de concessions, le règne des financiers et des "brasseurs d'affaires", si néfaste aux Industries nouvelles, et que lui soit substitué, pour le plus grand bien de la.collectivité, celui des Techniciens, dont notre pays, comme l'Italie, compte toute une pláiade, trop souvent tenue à distance parce que considérés, par ceux-là, comme trop clairvoyants et trop peu souples pour l'adoration du Veau d'Or.

\section{$*$}

Nota. - A l'heure où nous achevons la correction des épreuves de cet article, nous parvient - (hommage de notre estimé collègue, l'Ingénieur Santangelo) - un examplaire du magnifique ouvrage de ses camarades, les ingénieurs Baroni, Kambo, Granzotto et Marzo'i, d'édition récente, sur l'ouvrage qui nous occupe.

Nous regrettons vivement - l'envoi nous parvenant trop tard - de ne pouvo:r faire état, à leur place, de certains renseignements qu'il contient, et qui eûssent, à coup sûr, intéressé nos lecteurs ; comme, par exemple, le devis du dossier d'adjudication (qui prévoit l'emploi de mortiers bâtards) et l'état chronologique des formalités administratives (duquel ressort que le projet modificatif de l'ouvrage (comportant la superstructure à arches multiples), présenté au Génie civil en février 1922, n'avail pas encore reçu de cette Administration, son approbation officielle, le 1er décembre 1923 - jour de la catastrophe - ... (1).

(1) Malgré que cette Administration - conformément à une pratique déplorable mais courante, paraît-il, au-clelà des Alpes -fût d'accord avec le concessionnaire pour l'application de ce projet, et qu'elle en ait suivi régulièrement les travaux, il n'en résulte pas moins que sa responsabilité morale est sérieusement engagée dans cette affaire, du fait de l'inconcevable retard apporté par elle à l'approbation du projet ainsi exécuté entierement à titre officieux.
Malgré l'antorité indiscutée de ses auteurs - à laquelle nous rendons personnellement hommage - Iesquels ont cru pouvoir, en toute bonne foi à coup sûr, assumer la défense lechnique de la maison Vigano, il n'en résulte pas moins que ce document conslitue, pour celte Entreprise, un long plaidoyer "pro domo", qui n'apporte vraiment, dans l'affaire, aucume lumière de plus que les investigations déjà relatées.

A titre documentaire, el sans aucunement prendre position ni suivre nos éminents collegues sur ce lerrain, nous signalerons toutefois à nos lecteurs, une hypothèse nouvelle, à laquelle ont cru devoir s'arrêter longuement les auteurs de cetle brochure, et d'après laquelle la catastrophe serait uniquement due à $\mathrm{um}$ attentat criminel (!...), dans lequel ils veulent voir, comme délerminante de la rupture, la "cause instantanée "admise par les experts judiciaires.

En outre des constatations très détaillées qu'ils firent dans les ruines de la galerie de décharge el qui, selon eux - surloul, disent-ils, en raison de la rupture, un peu spéciale, des consoles supportant la passerelle - paraissent altester d'une explosion, survenue sur cette passerelle ou, pour le moins, contre la voute de la galerie, les avocats de la défense ont cru devoir prendre l'avis d'un ccrtain colonel Cugini, commandant le génie militaire du corps d'armée de Milan (1) ; el ce dernier, à son tour, en un docte rapport qui prend des allures "presque officielles " (2), conclut que "l'on doit admettre comme possible que la destruc"tion de la voûte et de la passerelle est la conséquence d'un "choc formidable, instantané " que, seule, peut avoir déterminé "l'explosion d'une forte charge d'explosifs..."

\section{Rapprochant de ces conclusions :}

$1^{\circ}$ La disparition, constatée sur le chantier, quelques jours avant la catastrophe, de 75 kilogs de dynamite;

$2^{\circ}$ La déposition, à la prison judiciaire de Cremona, du détenu Betli, prétendant qu'un de ses anciens compagnons de geôle nommé Della Matera, auteur de la destruction de la Centrale de l'Adamello, lui aurait déclaré que cette destruction n'était "qu'un commencement de représallles contre les fascistes du «Val Camonica pour les punir de leur attitude cruelle envers la "population ouvrière du Val (?...) et que "les préparatifs étaient "en cours pour faire sauter aussi celui du Gleno (3)";

Il faut convenir que ces coïncidences sont susceptibles de paraître troublantes et d'être exploitées par la défense de là Maison Vigano, en vue de laquelle, du reste, la contre-expertise et les dépositions ci-dessus ont été provoquées.

Quoi qu'il en soil, et quelle que soit la valeur que l'on puisse attribuer à cette hypothèse nouvelle d'un altentat criminel, elle ne saurait en rien annuler les conclusions du rapport des Experts, pas plus que celles qui terminent cette étude, ni même en atténuer la gravité, attendu que, même dans cette hypothèse, les responsabilités du concessionnaire et des Entrepreneurs n'en demeurent pas moins gravement engagées dans les malfaçons que nous avons relevées à leur charge, lesquelles par essence, prédisposaient l'ouvrage à une destruction plus ou moins prochaine, qui serait fatalement survenue en dehors même de toute provocation par une "cause instantanée".

J. B.

(1) Personnellement, nous sommes persuadé (et le regrettons pour eux) que l'autorité technique des contre-experts n'a rien gagné à s'entourer de parejl avis "militaire".

(2) Annexe no 54 du contre-rapport "des ingénicurs Baroni, Granzotto, Kambo et Marzoli.

(3) Annexes $n^{05} 52-53$ du contre-rapport des ingénieurs Baroni, Cranzotto, Kambo el Marzoli. 